\title{
List It: A Shopping List App That Influences Healthy Shopping Habits
}

\author{
Ifeoma Adaji \\ University of Saskatchewan \\ Saskatchewan, Canada \\ Ifeoma.adaji@usask.ca
}

\author{
Kiemute Oyibo \\ University of Saskatchewan \\ Saskatchewan, Canada \\ Kiemute.oyibo@usask.ca
}

\author{
Julita Vassileva \\ University of Saskatchewan \\ Saskatchewan, Canada \\ jiv@cs.usask.ca
}

\begin{abstract}
Unhealthy eating habits and a sedentary lifestyle have been identified as the two leading causes of obesity and weight gain in people. While there has been a lot of research on promoting active lifestyles, the area of influencing healthy eating habits is still under-researched. Because people will likely eat what they buy, it is imperative to influence people to shop for healthy foods in order for them to eat healthy foods. The use of a grocery list for planning one's shopping needs has been shown to influence healthy shopping habits among consumers. To contribute to ongoing research in the area of influencing healthy shopping behaviour, we introduce List It, an app that easily generates a shopping list for users from a list of various options while motivating the consumer to select healthy options to add to the list.
\end{abstract}

Healthy shopping habits, shopping list, influence strategy

\section{INTRODUCTION}

Over two billion adults and children worldwide are overweight or obese [3]. Many of these people suffer from serious diseases such as diabetes and heart-related diseases such as Ischaemic heart disease, which has been identified by the World Health Organisation as the number one cause of death worldwide ${ }^{1}$. Living an active life and eating healthy foods have been identified as the two major ways of improving one's health, reducing weight and preventing weight gain [2]. The last few years have seen an increase in research promoting active lifestyles such as the development of games that influence people to be more active [9],[5] and the development of fitness apps and devices such as the Fitbit ${ }^{2}$ that encourages people to move around and exercise more. However, more can be done in the area of persuading people to eat healthy, in particular, influencing consumers to shop for healthier foods in order that they will eat healthier foods. We hypothesize that if consumers are influenced to shop for healthy foods, they will likely eat healthy because people typically cook and eat what they buy or shop for. Thus, shopping for healthier foods could aid people in improving their health.

\footnotetext{
${ }^{1}$ http://www.who.int/news-room/fact-sheets/detail/the-top-10causes-of-death

${ }^{2}$ www.fitbit.com
}

One way consumers can be influenced to shop for healthy foods is by using a grocery list. The use of a grocery list has been identified as a useful tool in improving one's diet [4], [6] and decreasing one's body mass index especially for low-income households [4]. Dubowitz et al. [4] suggest that shopping with a grocery list may limit impulsive buying and could act as a "shield against the availability of unhealthy foods".

Writing a shopping list is one thing, but actually taking it to the store or not losing it is another challenge. Because people will likely take their mobile phones with them everywhere they go, in this position paper, we present the idea of a shopping list, List It, in the form of a mobile application. With List It, shoppers do not need to type out the items to be included in their shopping list, instead, they can simply select the products they want to include from a list of products. To further persuade consumers to shop for healthy foods, the calorie content of each product is visible in the shopping list app. Furthermore, consumers can add friends to their network and view the weekly statistics of the healthiness of their shopping lists compared to that of their friends.

\section{LIST IT MOBILE APPLICATION}

Research has shown that using a shopping list to plan one's shopping can improve one's diet and reduce their body mass index [4], [6]. List It is a 
shopping list mobile application that consumers can use to create a shopping list on the go. It allows shoppers to click items off a checklist and add such items to their shopping list instead of having to write them down one at a time or type them out manually. The app also informs shoppers of the calorie content of the items on the checklist so that the consumers can make an informed decision while adding products to their list. Furthermore, the app allows shoppers to become friends with other shoppers. By being friends with others, a consumer is able to compare the calorie content of their list to that of their friends. This way, they are motivated to add only healthy foods to their shopping list. In List It, products are grouped in categories, thus, a shopper can easily locate an item based on the category it falls under.

Over 4 billion people worldwide have mobile phones ${ }^{3}$. Because people use their phones very often [1], it is unlikely that consumers will leave home without their mobile phones. We thus developed List It as a mobile application that is compatible with both Android and iOS mobile devices. In the following section, we describe why we developed the app, the functionalities of List It, its architecture and implementation.

\subsection{The need for a shopping list app}

Although research has shown that the use of a shopping list can improve one's diet, we still sought to enquire from actual shoppers what healthy shopping habits they adopt when they go shopping. We carried out an online survey of 244 ecommerce shoppers. These participants were recruited through Amazon's Mechanical Turk, various news bulletins and social media. The study was approved by the ethics board of our university. Among other questions, we asked them to answer on a 7 point Likert scale, ( 1 being strongly disagree and 7 being strongly agree), how much they agree or disagree with the following statements:

\section{A: I make out a food list before I go shopping \\ B: I buy extra food items when I can buy them at lower prices \\ C: I check food ads in the newspaper before I go food shopping \\ D: I check food labels before purchasing a product for the first time \\ E: I plan menus before I go food shopping \\ F: I am the major food shopper for my family}

The average response from the respondents as shown in figure 2 indicates that the average participant agrees to use a shopping list to plan their shopping. This preliminary result gave rise to the idea of developing a shopping list app that consumers can use to plan their shopping needs.

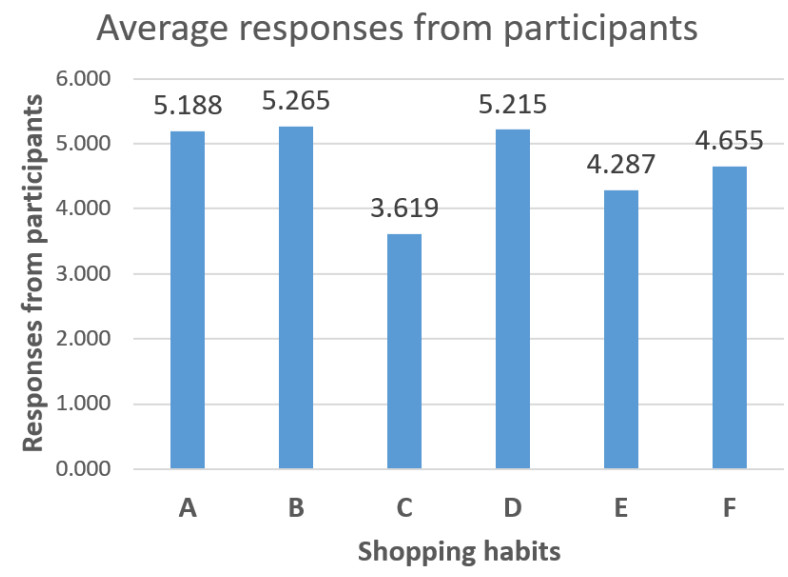

Figure 1: Average responses from participants about their shopping habits, $A=I$ make out a food list before I go shopping, $B=I$ buy extra food items when I can buy them at lower prices, $C=I$ check food ads in the newspaper before I go food shopping, $D=$ I check food labels before purchasing a product for the first time, E= I plan menus before I go food shopping, $F=$ I am the major food shopper for my family

\subsection{Using List It}

List It was designed to be easy to use by people of all ages and genders. Figure $2 \mathrm{~A}$ shows the landing page of the app. From the landing page, shoppers can check the available deals from the two major grocery retailers in Canada: Real Canadian Superstore and Walmart. From there, they can access the menu items (figure $2 \mathrm{~B}$ ) to decide what category of products they want to add to their list. For now, the app only has two categories of products: fruits and vegetables. In addition, from the menu, the shopper can view their current shopping list which they can easily update, login or sign up to the app, add friends or view the current leaderboard of friends.

If a shopper chooses to add fruits or vegetables to their shopping list, they will be presented with the fruits or vegetable screen as shown in figure $2 \mathrm{C}$ and $2 \mathrm{D}$ respectively. Both menu items display the calorie content of each product; this is to help shoppers in reaching an informed decision about the products they choose to add to their list.

The leaderboard as shown in figure $2 \mathrm{E}$ describes to the shopper how healthy a shopper's list is (usually the item in the leaderboard in bold) compared to that of their healthiest three friends. In this example, the current user's (Ify Adaji) shopping list is in the $12^{\text {th }}$ position in terms of healthiness, while the friend with the healthiest shopping list is John Nku.

\footnotetext{
${ }^{3}$ https://www.statista.com/statistics/274774/forecast-of-mobilephone-users-worldwide/
} 


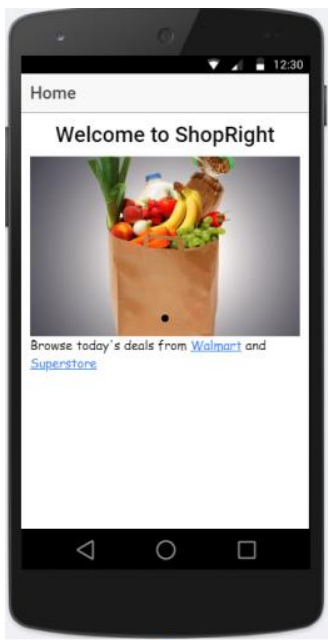

A
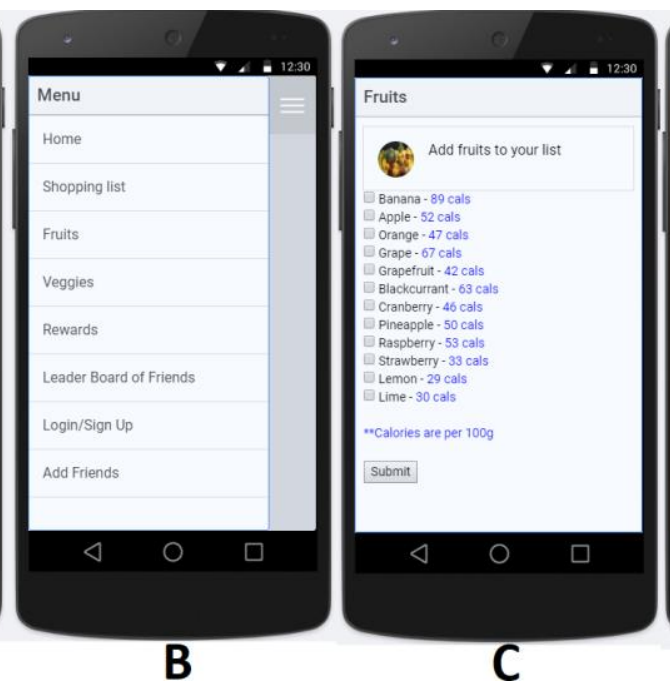
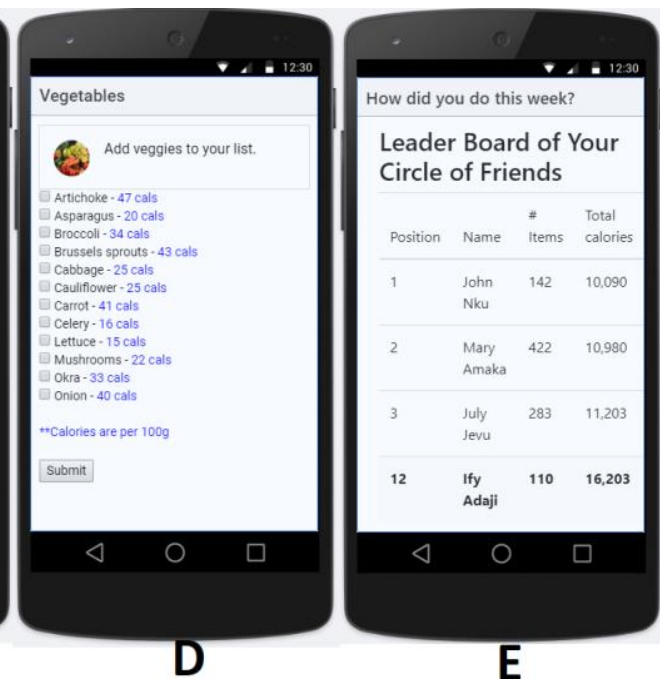

Figure 2: Some of the functionalities of List It app

\subsection{System architecture and implementation}

List It was developed using lonic ${ }^{4}$. Ionic is an open source development framework for hybrid mobile app development/ for web developers. Ionic allows developers build native applications that can run on any platform (including Android and iOS) using web development tools. We chose to use lonic because it is easy to use and it is cross-platform, across multiple devices.

\subsubsection{Persuasive strategies implemented}

In order to influence participation and usage of the app, we implemented some persuasive strategies that could bring about the change in behaviour we want. Persuasive strategies are human or computerbased communication designed to bring about a change in behaviour or attitude without coercion or force [10]. There are several commonly used strategies, some of which were adopted in List It. We present these in the following paragraphs.

\section{Rehearsal}

According to Oinas-Kukkonen [8], a system that allows its users to rehearse a target behaviour will likely be more persuasive. List It allows unregistered shoppers to create shopping lists. However, to use other functionalities such as adding friends, the shopper will have to create an account.

\section{Competition}

Humans naturally tend to compete with each other, thus creating an environment that fosters competition could make people adopt a target behaviour [8]. Leaderboards have been shown to encourage competition thereby bringing about the desired behaviour change [7]. We thus

\footnotetext{
${ }^{4}$ https://ionicframework.com/
}

implemented a leaderboard in our app as shown in figure $2 \mathrm{E}$ to encourage competition among consumers in the same circle of friendship. If a shopper such as Ify Adaji who is currently in $12^{\text {th }}$ position in terms of healthiness of her shopping list (figure 2E) sees her friend John Nku in the first position as being the person with the healthiest shopping list, Ify Adaji could be persuaded to only add healthier products to her list so that she could be at the top of the leaderboard.

\section{Self-monitoring and social comparison}

Systems that allow users to monitor and track their status or progress have been found to be more persuasive [8]. As shown in figure 2E, shoppers can monitor how healthy their shopping list is for the week. In addition, they are able to compare the healthiness or otherwise of their shopping list to that of others in their social circle.

\subsubsection{Benefits of List It over traditional shopping lists}

While most people create their shopping lists using pen and paper, List It does not require pen and paper but a mobile phone. Paper can get wet, torn, lost, or forgotten at home, however, a mobile phone will most likely not to get wet, torn or lost, neither will it likely be forgotten at home when a consumer goes to the store. In a case where a shopper forgets to write down an item on a paper shopping list, it is unlikely the shopper will remember to purchase such an item. On the other hand, because List It does not require the consumer to write anything, it is unlikely they will forget to include an item on their list. All that is required of the consumer is to check items off the list of products and these are added to their shopping list automatically. A quick run through of all items in a category will alert a consumer to any item(s) they might have forgotten to add to their shopping list. 
List It at a glance reveals to the shopper the number of calories that are in a food item before the shopper adds the product to their shopping list. This helps the consumer select healthier food options and it aids the shopper in the planning of healthier meals.

\section{EVALUATION}

Because List It is still in the development phase, we were unable to carry out a formal evaluation of the app. We, however, invited a few lab mates and friends to share their thoughts about the app with us. Note that this was not a formal evaluation of the app. The responses we got include the following:

- More products should be added to the app.

- The price of products from the two major retailers in the landing page of the app (Real Canadian Superstore and Walmart) could be included as it can help shoppers to decide on which of the grocery stores to shop at.

- The rewards could include virtual points or trophies that are displayed by each shopper's name in the leaderboard

- To eliminate a new registration process, the app should allow shoppers to use their login from existing platforms such as Facebook or Gmail.

List It is still in its very early phase. This paper aims to show proof of concept; the possibility of developing a shopping app that could be useful in planning the shopping of consumers with the aim of influencing healthier shopping habits.

\section{CONCLUSION AND FUTURE WORK}

Unhealthy eating habits and sedentary lifestyle have been identified as the leading causes of obesity and weight gain in people. In order to promote healthy eating habits, we propose to influence healthy shopping habits among consumers. The use of a grocery shopping list has been identified as one way that people are able to shop for healthy foods. To contribute to ongoing research in this area, we introduce List It, an app that creates a shopping list for consumers on the go. It uses various influence strategies such as competition, self-monitoring and social comparison.

List It is still in its infancy stage. In the future, we plan to integrate more features based on the feedback from users that are described in section 3. We also plan to carry out a formal evaluation approved by the ethics board of our university where real consumers will use our app and answer questions to determine if there are any changes in their shopping behaviour.

\section{REFERENCES}

[1] Billieux, J. et al. 2015. Can Disordered Mobile Phone Use Be Considered a Behavioral Addiction? An Update on Current Evidence and a Comprehensive Model for Future Research. Current Addiction Reports. 2, 2 (Jun. 2015), 156162. DOI:https://doi.org/10.1007/s40429015-0054-y.

[2] Bischoff, S. et al. 2017. Towards a multidisciplinary approach to understand and manage obesity and related diseases. Clinical nutrition. Volume 36, Issue 4 (2017), pp 917-938.

[3] Collaborators, T.G. 2015 O. 2017. Health Effects of Overweight and Obesity in 195 Countries over 25 Years. New England Journal of Medicine. 377, 1 (Jul. 2017), 1327.

DOI:https://doi.org/10.1056/NEJMoa161436 2.

[4] Dubowitz, T. et al. 2015. Using a Grocery List Is Associated With a Healthier Diet and Lower BMI Among Very High-Risk Adults. Journal of nutrition education and behavior. 47, 3 (2015), 259-64.

DOI:https://doi.org/10.1016/j.jneb.2015.01.0 05.

[5] Grimes, A. et al. 2010. Let's play!: mobile health games for adults. $n$ Proceedings of the 12th ACM international conference on Ubiquitous computing (2010), 241-250.

[6] Hersey, J. et al. 2001. Food shopping practices are associated with dietary quality in low-income households. Journal of Nutrition Education. 33, (2001), S16-S26.

[7] Jin, Y. et al. 2016. A Little Competition Never Hurt Anyone's Relevance Assessments. GamifIR@ @IGIR (Pisa, Italy, 2016), 29-36.

[8] Oinas-Kukkonen, $\mathrm{H}$. and Harjumaa, M. 2008. A systematic framework for designing and evaluating persuasive systems. Persuasive technology. Springer. 164-176.

[9] Orji, R. et al. 2017. Towards Personalitydriven Persuasive Health Games and Gamified Systems. Proceedings of the 2017 $\mathrm{CHI}$ Conference on Human Factors in Computing Systems - CHI '17 (New York, New York, USA, 2017), pp 1015-1027.

[10] Simons, H.H.W. and Jones, J. 2011. Persuasion in society. Taylor \& Francis. 